\title{
Cyber-Physical System Modeling Using a Case Study
}

\author{
Sara Mallah $^{1,2(\bowtie)}$, Khalid Kouiss ${ }^{3}$, Oualid Kamach ${ }^{1,2,3}$, \\ and Laurent Deshayes ${ }^{1,2,3}$ \\ ${ }^{1}$ ILO, University Mohammed 6 Polytechnic, Ben Guerir, Morocco \\ sara.mallah@um6p.ma \\ 2 LTI, ENSA, Tangier, Morocco \\ ${ }^{3}$ SIL, Blaise-Pacal University of Clermont Ferrand, Aubière, France \\ khalid.kouiss@ifma.fr
}

\begin{abstract}
The fad in today's market for customer-specific products pushed the industry to renew itself and drive value creation initiative. In fact, companies are concerned not only about selling the product as a function, but also about selling the value as a solution. It is reasonable to think that the creation of these new business models involve building flexible manufacturing facilities, digitizing and integrating inter and intra-company systems into one intelligent data management structure which allow physical and software components to interact with each other in a myriad of ways that change with context, in spite of the different spatial and temporal scales they operate on. This synergic interaction can be fulfilled by accomplishing an industry 4.0 environment that aims to transcend mechatronic systems and move to cyber-physical systems (CPS).

In this paper, we present our methodology to model CPS. The results show promising research opportunity for implementing CPS in industry.
\end{abstract}

